\title{
Erratum to: Visual working memory always requires attention
}

\author{
Candice C. Morey • Malte Bieler
}

Published online: 9 January 2013

(C) Psychonomic Society, Inc. 2012

\section{Erratum to: Psychon Bull Rev \\ DOI 10.3758/s13423-012-0313-z}

An error in the script used to process the data caused the values in Figs. 2 and 3 and some values in our analysis to be incorrect. In the full analysis of binding versus color features, the effect of retention condition in the first ANOVA reported should be $F(2,196)=42.91, M S E=0.02, \eta_{G}^{2}=.12$. The main effect of retention interval in the full analysis of shape features versus binding should be $F(2,196)=43.33, M S E=0.02, \eta_{G}^{2}=.13$; restricted to only the two-item trials, this effect should be $F(2$, 196) $=29.62, M S E=0.04, \eta_{G}^{2}=.11$. Each of these effects remains statistically significant, with $p$ s $<.05$.

All of the effects that were originally reported as null remained null after reanalysis.

We conducted new Bayes factor ANOVAs upon discovering these errors, this time sampling 100,000 iterations for better precision. For the binding versus color analysis, the model including main effects of group and retention condition yielded the highest Bayes factor $(B F=112.82)$, followed by the model including both main effects and their interaction $(B F=106.19)$. Comparing these two models directly, the evidence provided in the data for the simpler model is greater by a factor of 761:1. For the binding versus shape analysis, the model with the highest Bayes factor included only the retention condition effect $(B F=$ 98.35), followed by the model including both main effects $(B F=96.62)$. The best model including the interaction yielded a Bayes factor of 88.54. Comparing these models, the retention condition effect model was preferred to the two-effect model by a factor of $6: 1$, but both of these models were preferred to the best model including the interaction by factors of at least 3,218:1. These inferential outcomes are the same as those in the original report; our conclusions therefore remain unchanged.

We are grateful to Klaus Oberauer for asking about irregularities between the means depicted in Fig. 2 and the collapsed condition means reported in the text, which led to the discovery of these errors.

The online version of the original article can be found at http://dx.doi.org/ 10.3758/s13423-012-0313-z.

C. C. Morey $(\bowtie) \cdot$ M. Bieler

Experimental Psychology, University of Groningen,

Grote Kruisstraat 2/1,

9712TS Groningen, The Netherlands

e-mail: c.c.morey@rug.nl 
Fig. 2 Discrimination accuracy for bound probes and color feature probes under each of the encoding conditions. Error bars are standard errors of the mean, with the Cosineau-Morey correction applied (R. D. Morey, 2008)

Fig. 3 Discrimination accuracy for bound probes and shape feature probes under each of the encoding conditions. Error bars are standard errors of the mean, with the Cosineau-Morey correction applied (R. D. Morey, 2008)
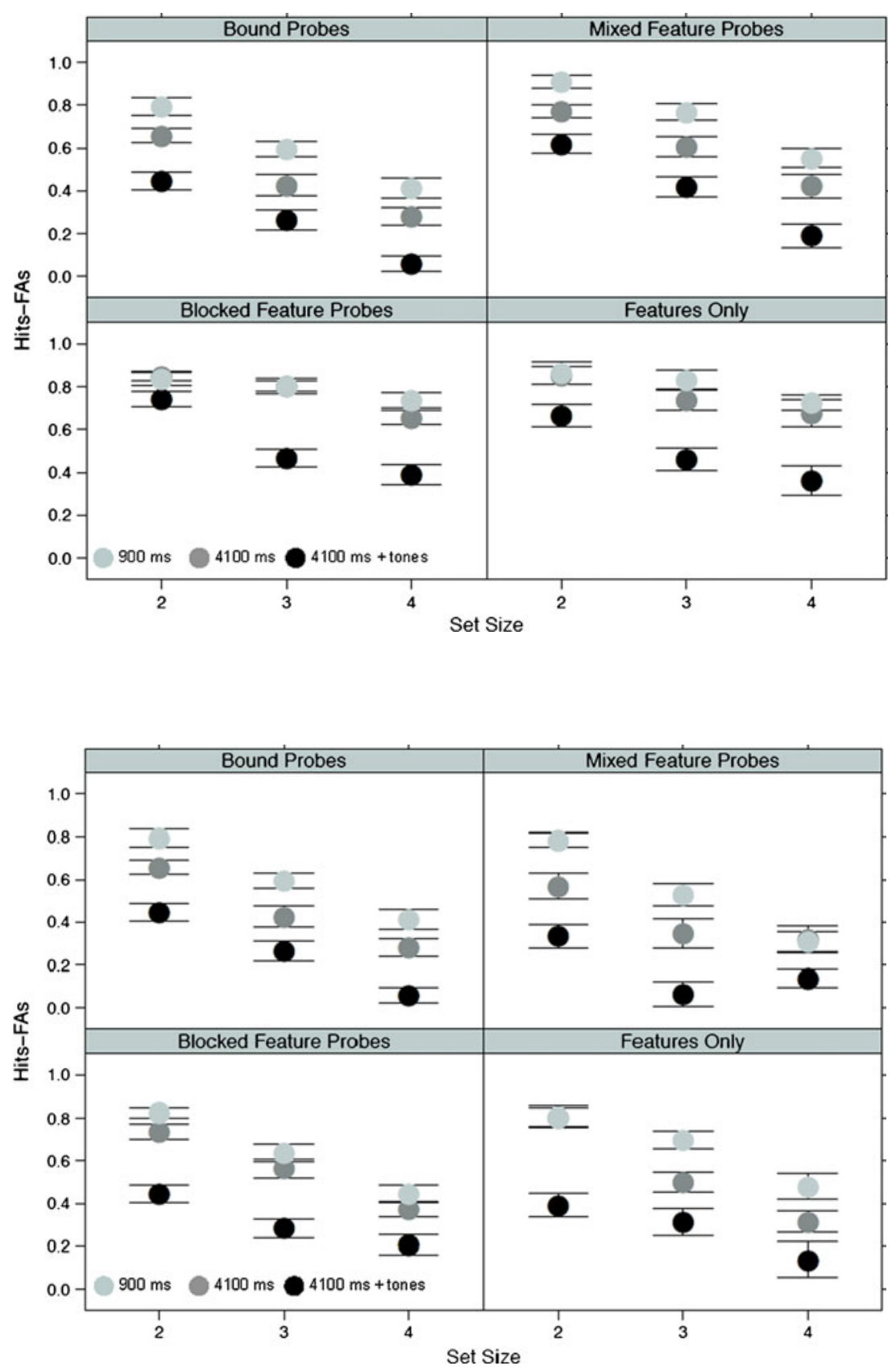